\title{
Los recursos educativos que los profesores de Secundaria estiman necesarios para desarrollar procesos educativos inclusivos
}

\author{
Teachers' perception about the necessary \\ educational resources to develop inclusive \\ processes
}

\begin{abstract}
Resumen
El presente artículo se ha elaborado a partir de una investigación llevada a cabo por profesores del departamento de Análisis Social de la Universidad Carlos III de Madrid y financiada por la Fundación ONCE. En ella se han utilizado encuestas y entrevistas en profundidad para conocer las necesidades y carencias del profesorado de secundaria para desarrollar procesos educativos inclusivos. La información recogida refleja que tanto los profesores como los centros educativos no disponen, de manera homogénea, de los recursos necesarios para que todos los estudiantes puedan educarse de manera conjunta. Disponer de políticas y proyectos educativos inclusivos, un centro adaptado arquitectónicamente, materiales adaptados a cada discapacidad, apoyos a la docencia y una disminución de la ratio profesor/ alumno son recursos que facilitan la inclusión de los alumnos con discapacidad. No obstante, la ausencia de estos recursos genera exclusión y crea barreras para desarrollar un proceso inclusivo educativo.
\end{abstract}

\section{Palabras clave}

Recursos educativos, estudiantes con discapacidad, informe evaluativo, profesionales de apoyo, adaptación del material, recursos tecnológicos, adaptación del centro, ratio profesor/ alumno, políticas y proyectos educativos.

\begin{abstract}
This paper is the result of a research conducted by the Department of Social Analysis of the Universidad Carlos III of Madrid and funded by the ONCE Foundation. Surveys and in depth interviews were used to determine the needs and shortages secondary school teachers experience in the education students with disabilities. The data collected and the testimonies of the teachers reflect that schools don't have the resources needed for education all students at the same school, in the same classroom and at the same time. The architectural adaptation of the buildings, the materials and technology used, the of support teachers avaible and a high student-teacher ratio facilitate the development of inclusive educational processes. Nevertheless, the absence of these resources generates exclusion and creates barriers to develop these processes.
\end{abstract}

\section{Keywords}

Educational resources, students with disabilities, assessment report, support teachers, adaptation of the educational material, technological resources, architectural adaptation, student/teacher ratio, educative policies.

\section{Vicente Díaz Gandasegui <vdgandas@polsoc.uc3m.es> \\ Profesor Ayudante Doctor. Departamento de Análisis Social. Universidad Carlos III de Madrid.}

Fernando Caballero Méndez $<$ fcaballe@polsoc.uc3m.es>

Profesor Asociado. Departamento de Análisis Social. Universidad Carlos III de Madrid.
Para citar:

Díaz Gandasegui, V. y Caballero Méndez, F. (20I4): "Los recursos educativos que los profesores de Secundaria estiman necesarios para desarrollar procesos educativos inclusivos". Revista Española de Discapacidad, 2 (I): 97-II3.

<http://dx.doi.org/IO.5569/23405 I04.02.01.05>

Fecha de recepción: I7-O2-20I4 Fecha de aceptación: I6-06-20I4 


\section{Introducción}

En el marco de una investigación sobre las necesidades y demandas formativas de los profesores de secundaria (E.S.O, FP, Bachillerato, ciclos formativos y PCPI) desarrollada por el departamento de Análisis Social de la Universidad Carlos III de Madrid y financiada por la Fundación ONCE para el año 20I3, se han analizado también los recursos educativos, materiales y humanos que los profesores estiman necesarios para desarrollar una escuela inclusiva. ${ }^{\mathrm{I}}$

Además de que el profesorado reciba formación, una condición indispensable para afrontar un proceso inclusivo con éxito, es fundamental que los docentes cuenten con unos recursos educativos que permitan que los alumnos con discapacidad se eduquen junto al resto de sus compañeros en el centro al que acudirían si no tuvieran discapacidad, facilitando así su presencia, aprendizaje y participación (Porter, I997; Echeita Sarrionandia, 2009; Ainscow, Booth y Dyson, 2006).

Para ello, todos los centros educativos deben estar preparados para recibir a todos los alumnos. De esta manera se evita que los profesores tengan que improvisar cuando se encuentran en sus aulas a alumnos con discapacidad y, simultáneamente, que los estudiantes sufran procesos de segregación, siendo derivados a itinerarios educativos alternativos o, en última instancia, trasladados a otro centro.

No obstante, es importante tener en consideración que los recursos educativos inclusivos tampoco garantizan que se produzca un proceso inclusivo. En el fondo, como indica Mel Ainscow (1995: 37), la diferencia son las personas y como éstas sepan gestionar y

I. La investigación "Las necesidades formativas de los docentes en la educación de estudiantes con discapacidad" (Caballero Méndez y Diaz Gandasegui, 20I4) ha sido publicada por la Fundación ONCE. utilizar los recursos educativos. Por ello, nuestra investigación se ha centrado en el profesorado, recabando información sobre los recursos disponibles en sus centros y, también, las reflexiones y opiniones sobre las implicaciones que tiene para los alumnos con discapacidad y su labor docente la presencia o ausencia de estos recursos.

Los docentes han identificado y analizado una serie de barreras y elementos facilitadores para desarrollar procesos educativos inclusivos en sus centros de secundaria. La información que han aportado los profesores nos ha servido también para reflexionar sobre la relevancia y trascendencia que tienen estos recursos educativos para afrontar un proceso inclusivo con garantías.

\section{Metodología}

Las muestras utilizadas tanto para la fase cuantitativa como cualitativa de la presente investigación fueron diseñadas teniendo en cuenta los datos proporcionados por el Ministerio de Educación, Cultura y Deporte y el Instituto Nacional de Estadística sobre el profesorado de secundaria en España.

Para la realización de la encuesta se utilizó un muestreo aleatorio estratificado para 250 individuos, determinando los distintos estratos con una afijación proporcional con las variables que fueron consideradas relevantes para la investigación: el ámbito (rural y urbano) y el tipo de centro (público, privado y concertado).

\begin{tabular}{|l|c|c|c|}
\hline & Público & Concertado & Privado \\
\hline Rural & 29 & 20 & 4 \\
\hline Urbano & 108 & 73 & 16 \\
\hline
\end{tabular}

Los encuestados fueron seleccionados aleatoriamente y se les contactó mediante un 
correo electrónico enviado a las secretarías de los centros educativos; se recibieron respuestas de centros de todas las comunidades autónomas excepto de la Región de Murcia.

Se diseñó un cuestionario online autorrellenable consistente en 24 ítems, de los cuales cuatro eran filtros que daban acceso a preguntas específicas. Además, el cuestionario incluyó ocho escalas Likert con cuatro opciones de respuesta cada una, las cuales añadieron datos sobre actitudes y opiniones a la información sobre hechos y comportamientos que se recabó con el resto de preguntas.

Para analizar la información recogida mediante las encuestas se utilizó el software SPSS I7.O.

Para la realización de las entrevistas en profundidad se diseñó una muestra de 24 profesores, respetando, proporcionalmente, los criterios de muestreo utilizados para la metodología cuantitativa.

\begin{tabular}{|l|c|c|c|}
\hline & Público & Concertado & Privado \\
\hline Rural & 3 & 2 & 0 \\
\hline Urbano & 11 & 7 & 1 \\
\hline
\end{tabular}

Las entrevistas fueron semi-estructuradas, lo que permitió, a partir de un guion básico, introducir y variar las preguntas y los temas, facilitando que el entrevistado tuviese libertad en las respuestas y el discurso. Las entrevistas aportaron un componente descriptivo y aclaratorio sobre los recursos educativos que están presentes o ausentes en los centros educativos en los que trabajan. Así, se compaginó la información aportada por las encuestas con la reflexión libre y espontánea de las entrevistas, que permitió la indagación y la aclaración sobre las respuestas recibidas en los cuestionarios.

Los entrevistados fueron seleccionados de manera estratégica para que cumpliesen los criterios establecidos; las entrevistas en profundidad fueron concertadas mediante llamadas telefónicas a los centros educativos. Las entrevistas fueron realizadas en la Comunidad de Madrid, Castilla- La Mancha y Extremadura.

Una vez transcritas las entrevistas se utilizó la herramienta informática Nudist Nvivo 7.0, que permitió analizar el discurso de los entrevistados.

\section{Análisis de los recursos educativos para facilitar la inclusión}

Los recursos que facilitan u obstaculizan la inclusión de los estudiantes con discapacidad son aquellos elementos que utiliza el profesorado y aquellas instalaciones que poseen los centros educativos que pueden "condicionar positiva o negativamente la presencia, el aprendizaje o la participación de determinados alumnos en la vida escolar" (Casanova Rodríguez y Cabra de Luna, 2009: 68). Los recursos educativos que se analizan en este artículo son diferentes piezas que no garantizan por sí solas la existencia de un proceso educativo inclusivo, pero sí son imprescindibles para que éste tenga lugar.

La formación y conocimientos de los docentes son fundamentales para que los estudiantes puedan obtener una experiencia educativa inclusiva, pero existen otros recursos que no dependen directamente de los profesores, aunque son necesarios para que se desarrollen dichos procesos.

La existencia de estos recursos depende, en gran medida, de las políticas y proyectos educativos que desarrolle el centro. De este modo, una política educativa que favorezca la inclusión de los estudiantes con discapacidad debe ser el principio desde el que se articulen todas las medidas educativas que tome el centro. De lo contrario, si no existen políticas educativas que proporcionen los recursos educativos necesarios y no se crea un plan de acción para que estos recursos estén disponibles cuando sean precisos 
y en el momento en que se requieren, se crea ineficacia (Ferrandis, Grau y Fortes, 2010: 25).

Los profesores, objeto de estudio de esta investigación, son los principales mediadores entre las políticas que promulga el centro educativo y los alumnos; por ello es importante conocer la percepción que ellos tienen sobre la aplicación y existencia de estas políticas, especialmente su implicación e interpretación de la cultura del centro en el que imparten docencia (Vallejo Ruiz y Bolarín Martínez, 20I I). En este sentido, el $65 \%$ de los profesores encuestados declararon que sus centros poseen un proyecto educativo para atender las necesidades de los estudiantes con discapacidad, mientras que el $26 \%$ de los docentes declara que su centro no dispone de tal proyecto y que las políticas inclusivas dependen de las iniciativas que desarrolle cada profesor. Únicamente un $2 \%$ de los profesores encuestados indicó que su centro prefiere derivar a los estudiantes con discapacidad a centros especializados (Gráfico I).

La existencia de políticas para atender a las necesidades de estudiantes con discapacidad está relacionada con el tipo de centro educativo. Así, el $74 \%$ de los profesores encuestados de centros públicos, frente al $59 \%$ de los concertados y el $30 \%$ de los privados, indicaron que su centro poseía un proyecto educativo para atender las necesidades de estos estudiantes con discapacidad.

Pese a que en algunos casos la presencia de alumnos con discapacidad es determinante para desarrollar proyectos educativos inclusivos, en las entrevistas en profundidad hemos podido constatar cómo, en la mayoría de los casos, la existencia de un proyecto educativo para atender a los estudiantes con discapacidad es el factor que facilita que estos alumnos puedan estar presentes en las aulas junto al resto de sus compañeros. Este es un factor que probablemente explica la desigual distribución de estudiantes con discapacidad en centros de carácter público, concertado y privado. Los datos proporcionados por el Ministerio de Educación Cultura y Deporte (Tabla I) indican que la proporción de estudiantes con discapacidad es diferente dependiendo del tipo de centro educativo.

\section{Gráfico 1. Distribución del profesorado en función de cómo actúa el centro en el que trabaja con respecto a los estudiantes con discapacidad}

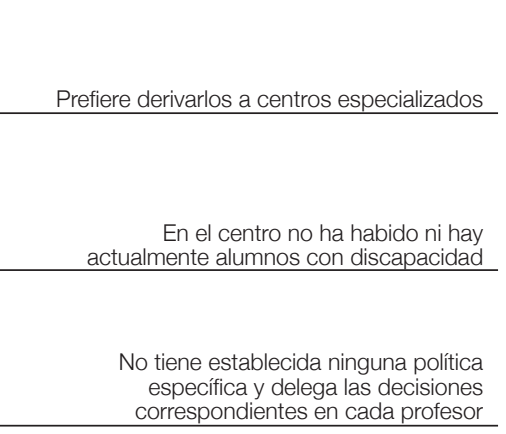

Posee un proyecto educativo para atender las necesidades de estos estudiantes

\section{2,0}
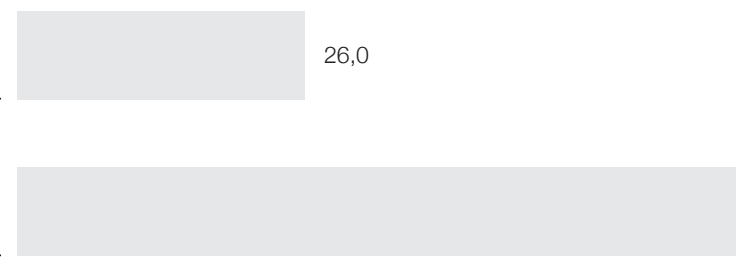

Fuente: Elaboración propia. 


\begin{tabular}{l}
\hline $\begin{array}{l}\text { Tabla 1. Porcentaje de alumnado con } \\
\text { necesidades educativas especiales respecto } \\
\text { al total de alumnado (Enseñanza Secundaria } \\
\text { Obligatoria) }\end{array}$ \\
\begin{tabular}{|l|c|}
\hline Tipo de centro educativo & $\%$ \\
\hline Públicos & 2,2 \\
\hline Privados-Enseñanza Concertada & 1,8 \\
\hline Privados-Enseñanza no Concertada & 0,2 \\
\hline
\end{tabular}
\end{tabular}

Fuente: MECD. "El alumnado con necesidades específicas de apoyo educativo. Curso 20I0-20I I".

La presencia de un proyecto educativo para atender a los alumnos con discapacidad en el centro afecta a muchos de los recursos que favorecen la inclusión de estos estudiantes. Los profesores que pertenecen a centros con un proyecto educativo inclusivo son los que, en mayor proporción, cuentan con informes evaluativos de los alumnos, más recursos materiales y tecnológicos, más apoyos de profesionales especialistas y en los que los centros están más adaptados.

Consecuentemente, hemos identificado, teniendo en consideración la experiencia, necesidades y carencias relatadas por los profesores que han participado en nuestra investigación, que los recursos que los docentes estiman necesarios para desarrollar un proyecto educativo inclusivo con éxito y garantías son: la presencia de informes evaluativos, la adaptación arquitectónica del centro, la disponibilidad de recursos materiales y tecnológicos que puedan ser utilizados por todos los estudiantes, la coordinación con profesores de apoyo y la modificación de la ratio profesor/alumno.

\subsection{Informe educativo}

El informe educativo es una herramienta fundamental para que los docentes puedan tener acceso a información relevante sobre el alumno, identificar sus necesidades y, por otro lado, disponer y diseñar un plan de enseñanza que se adapte al estudiante y al resto de compañeros.
Como señaló una de las docentes entrevistadas durante la investigación:

"El diagnóstico va unido a una determinación de necesidades y a una provisión de recursos con los cuales, de alguna manera, se está señalando una dificultad, pero también se están proviniendo una serie de recursos para trabajar esa dificultad" [V. M: Profesora de centro público urbano]

El informe es un documento con una doble naturaleza psicopedagógica y administrativa que ha de servir, en última instancia, para que el alumno con discapacidad pueda desarrollar su educación en las mejores condiciones posibles (MEC, I996: I 56) y que facilita que el profesorado pueda diseñar una metodología y actividades adecuada para todos los alumnos. En este sentido, el informe psicopedagógico en el sistema educativo español "se trata de una propuesta de organización de la información claramente abierta y flexible que no tiene por qué traducirse directamente en un modelo de informe estandarizado” (Téllez Gallego, 20ı0: 6).

Sin embargo, pese al potencial que posee el informe evaluativo para ayudar a todos los participantes en el proceso educativo a desarrollar procesos inclusivos, en el presente y el pasado reciente "ha consolidado un sistema tolerante con la exclusión, permisivo con mecanismos que generan exclusión" (García Pastor, 2005: I93). Esto se debe a que, frecuentemente, ha sido empleado para etiquetar a los estudiantes en unas categorías rígidas, lo que lleva a que se reduzcan las expectativas que los profesores tienen sobre los alumnos, desrresponsabilizándose de la situación de estos (Ainscow, I995: 28; Booth y Ainscow, 2002: 20; Sandoval Mena, Simón Rueda y Echeita Sarrionandia, 20I 2: I25) o, como indica Concepción García Romero, (2006: 52) se ha convertido en una fórmula "para calmar conciencias y descansar responsabilidades”. En este sentido, Miguel Ángel Parrilla Latas explica que:

"En el contexto escolar se desarrollan formas diversas de exclusión que, por haber sido naturalizadas a lo largo de la historia 
educativa, pasan desapercibidas y son consideradas parte del hacer tradicional de las instituciones. Es el caso, por ejemplo, de las bondades atribuidas a las etiquetas como instrumento para determinar el tipo más adecuado de educación para los alumnos; el uso de espacios e itinerarios especiales basados en modelos paliativos y terapéuticos que sitúan a los alumnos en la periferia académica" (Parrilla Latas, 2009: II 3 ).

La información que se genera sobre los alumnos debe tener la finalidad de ayudar y apoyar a los estudiantes con discapacidad únicamente cuando sea necesario, evitando un proceso de etiquetaje por el que se podría discriminar a determinados estudiantes en base a criterios desafortunados de "normalidad" fundamentados en una forma específica de acceder al conocimiento. El informe debe servir para determinar cómo es cada estudiante, sin que suponga la segregación de los alumnos dentro o fuera del aula. En este sentido, la manera en que se utilice el informe evaluativo por parte de los profesionales del centro puede suponer un recurso que facilite la educación de alumnos con discapacidad pero, también, puede constituirse en un obstáculo para que los estudiantes con necesidades educativas diferentes al resto puedan progresar (Casanova Rodríguez, 20I I: I 5 ).

El informe es, en definitiva, un instrumento complejo que debe ser elaborado por especialistas, pero en el que deben colaborar los profesores y personal de apoyo que conozcan al estudiante y tengan constancia sobre los recursos que se encontrará el estudiante en el centro. El informe debe atender a las necesidades del estudiante y las posibilidades de respuesta a esas necesidades que puede prestar el centro educativo, convirtiéndose éste en un protagonista activo del proceso inclusivo (Alonso Parreño y Araoz SánchezDopico, 20I I). El contexto en el que se educa el estudiante es un agente explicativo de las dificultades de aprendizaje que éste se encontrará y, por lo tanto, "hay que tener en consideración la interacción entre las características propias del alumnado y las de la acción educativa" (Ferreira, 2013: 5). Por consiguiente, es fundamental, identificar los obstáculos y las limitaciones que encuentra el estudiante atendiendo a los elementos materiales y humanos y, también, aludiendo a cuestiones intangibles que se refieren a los contextos ambientales, es decir, las políticas educativas, la cultura del centro y su organización (Echeita Sarrionandia, 2007: I I 2; Ainscow, I995: 27).

La existencia de un informe psicopedagógico es un recurso esencial para que el centro disponga de los recursos necesarios que requiere el alumnado, pero, pese a ello, el $25 \%$ de los docentes encuestados que ha tenido experiencia con estudiantes con discapacidad declaró que su centro no dispone de informes estandarizados (Gráfico 2).

El procedimiento más habitual para obtener información sobre los estudiantes con discapacidad entre los profesores encuestados es a través del personal especializado del centro educativo $(60 \%)$ (Gráfico 3 ).

En cuanto al tipo de centro, se observa que, mientras en los centros públicos la información proviene del personal especializado y del informe que facilita el centro educativo al que pertenencia el estudiante, en los centros privados la información es transmitida, en mayor proporción, por otros profesores del centro, por el diagnóstico del propio docente o por la familia del estudiante. Probablemente, esto se debe a que los centros públicos y, en menor medida los concertados, reciben la información por vías oficiales, siguiendo los protocolos establecidos, mientras que en los centros privados las fuentes de información son más informales y familiaristas (Tabla 2).

El momento en que los profesores reciben la información sobre los alumnos es indicador del tiempo que tuvieron para preparar la metodología, actividades y materiales apropiados para desarrollar el curso. Es significativo que el $38 \%$ de los profesores declararon que recibieron la información al comienzo del curso, mientras que el $5 \%$ fueron informados una vez había comenzado el curso. En este sentido, uno de los profesores entrevistados expresó el inconveniente que 
Fuente: Elaboración propia.

Gráfico 3. Distribución del profesorado en función de quién o quiénes le transmitieron la información sobre los estudiantes con discapacidad

El personal especializado del centro educativo

Otros profesores del centro

Orespresos de

El diagnóstico lo realizó el propio docente

Otros estudiantes 1

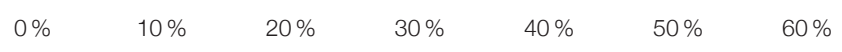

Fuente: Elaboración propia.

Tabla 2. Distribución del profesorado en función de procedencia del informe educativo, por tipo de centro

\begin{tabular}{|l|c|c|c|}
\hline Procedencia del informe educativo & Público & Concertado & Privado \\
\hline Del centro educativo al que pertenecía el estudiante en el curso anterior & $42 \%$ & $35 \%$ & $20 \%$ \\
\hline Del personal especializado del centro educativo & $66 \%$ & $50 \%$ & $50 \%$ \\
\hline De otros profesores del centro & $27 \%$ & $20 \%$ & $40 \%$ \\
\hline El diagnóstico lo realizó el propio docente & $11 \%$ & $11 \%$ & $30 \%$ \\
\hline De la familia del estudiante & $14 \%$ & $48 \%$ & $70 \%$ \\
\hline De otros estudiantes & $1 \%$ & $2 \%$ & $0 \%$ \\
\hline
\end{tabular}

Nota: El total es superior al I00\% por tratarse de una pregunta que admite más de una respuesta. Fuente: Elaboración propia. 
supuso desconocer las necesidades de una alumna hasta el mismo momento en que comenzó el curso:

“...la forma de enterarnos fue el primer día de clase, pues hacer adaptaciones ...claro, si a ti el centro te avisa y tienes quince días para mentalizarte, para pensar como adaptas un poquito las prácticas, la metodología de clase pues mucho mejor, pero así de repente era una situación complicada para todos..." [D. L: profesor de centro público, Urbano, FP].

\subsection{Adaptación del centro}

El primer recurso que se debe tener en consideración en el informe educativo es la capacidad del centro para acoger a todo tipo de alumnado, es decir, la adaptación arquitectónica de sus instalaciones. Si se quiere evitar la exclusión de determinados estudiantes todos los centros deberían estar adaptados a todos los alumnos. Es importante tener en consideración que la exclusión no solo tiene lugar en las aulas sino que también, y en primer lugar, ocurre cuando los centros presentan obstáculos para que ciertos estudiantes accedan y se desplacen por ellos.

Únicamente el $37 \%$ de los docentes manifestó que sus centros están adaptados, aunque es sorprendente que solo el $2 \%$ declaró que la falta de adaptación impidió la matriculación de alumnos con discapacidad (Tabla 3 ).

\begin{tabular}{l}
\hline $\begin{array}{l}\text { Tabla 3. Distribución del profesorado en función } \\
\text { de si el centro en el que imparte docencia está } \\
\text { adaptado y dispone de recursos suficientes para } \\
\text { que cualquier estudiante pueda utilizarlo }\end{array}$ \\
\begin{tabular}{|l|c|}
\hline Lo desconoce & $9 \%$ \\
\hline $\begin{array}{l}\text { No, aunque no ha impedido que pueda } \\
\text { matricularse cualquier estudiante }\end{array}$ & $51 \%$ \\
\hline $\begin{array}{l}\text { No. En algunos casos ha impedido } \\
\text { la matriculación de alumnos con } \\
\text { discapacidad }\end{array}$ & $2 \%$ \\
\hline Sí & $37 \%$ \\
\hline
\end{tabular}
\end{tabular}

Fuente: Elaboración propia.
Estos datos revelan un tipo de exclusión más velada, menos visible, pero no por ello menos importante, que ocurre cuando los estudiantes con discapacidad pueden acceder al centro educativo aunque no pueden participar en las actividades sociales y educativas en las mismas condiciones que sus compañeros (Booth y Ainscow, 2002: 22). Algunos de los profesores entrevistados, pese a ser conscientes de las limitaciones que supone la falta de adaptación del centro, parecen desconocer la importancia de que la inclusión de los alumnos con discapacidad no se circunscriba exclusivamente al aula:

"con sus limitaciones, evidentemente, a la hora de salir al patio pues los chicos con discapacidades motóricas no suelen salir, suelen quedarse en el hall, pero siempre suelen quedarse con dos, tres amigos que tienen y aunque las normas impiden que estén durante el recreo dentro del edificio pues, evidentemente, eso no reza para que lo hagan estos chicos ni los amigos que les acompañan, o sea, que en ese sentido perfecto..." [J.M.C: Profesor de centro público urbano].

La adaptación arquitectónica parcial y, por lo tanto, insatisfactoria afecta a los alumnos pero también a los docentes, que deben modificar las actividades educativas. Uno de los profesores entrevistados relató el reto que implica para docentes y alumnos la falta de adaptación arquitectónica de su centro:

\footnotetext{
"En el exterior sí que tenemos rampas, pero nada más. Por ejemplo, para bajar a los patios, a las canchas, no hay rampas, por ponerte un ejemplo. Son escaleras todo, a mí me gustaría que el centro estuviera adaptado, pero es que no hay posibilidades económicas..." [J. L: Profesor de centro concertado urbano].
}

\subsection{Recursos materiales y tecnológicos}

"Los recursos y materiales didácticos son todo el conjunto de elementos que el profesor utiliza, o puede utilizar como soporte, complemento o ayuda en su tarea docente" (Díaz Lucea, I996, 42). Siempre que sea posible, se deben utilizar 
los mismos materiales y tecnología para todos los estudiantes, aunque tengan diferentes niveles de habilidad. No obstante, cuando no es posible utilizar esta estrategia, una adecuada adaptación y diseño del material académico facilita que todos los estudiantes puedan realizar las mismas actividades en el mismo espacio y tiempo, evitando que los estudiantes con discapacidad se sientan infravalorados por realizar actividades diferentes o con menor dificultad (Trujillo Torres, Torres González, Hernández Fernández, 2OII: 84).

El 50\% de los docentes encuestados manifestaron que la falta de herramientas adecuadas a los perfiles de los alumnos fue una de las carencias que se encontraron cuando impartieron docencia a estudiantes con discapacidad. Además, el $62 \%$ de los profesores indicaron que les gustaría adquirir conocimientos para conocer los materiales que deben utilizar y cómo hacerlo cuando, entre los alumnos, hay estudiantes con discapacidad.

La falta de adaptación del material puede causar que los alumnos se sientan aislados, pese a encontrarse dentro del aula, debido a que no realizan la misma actividad que sus compañeros. Una de las profesoras entrevistadas describía la situación en la que se encontraba un alumno con discapacidad auditiva en su asignatura de inglés:

“Realmente él no trabaja inglés, sino que en las horas que tiene mi asignatura, que son cinco a la semana, me facilitan materiales donde este chico trabaja las instrumentales" [E.M: Profesora de centro público rural].

No obstante, uno de los profesores entrevistados nos relató la importancia que tuvo en su experiencia con estudiantes con discapacidad visual la adaptación del material para que todos los estudiantes pudieran realizar las mismas actividades:

“Antes del principio de curso dábamos la lista de libros y la ONCE se encargaba de pasarlos a braille. Entonces, para septiembre, octubre, noviembre, cuando se podía, pues ya estaban los libros de texto hechos en braille [...].
Los mapas en relieve en sociales, pues eran absolutamente distintos que los mapas gráficos de los chavales. Nosotros pedíamos que nos fabricasen, bueno la ONCE normalmente ya tenía fabricados mapas táctiles en relieve de plástico. Y los chicos cuando estábamos hablando de África pues estaban tocando y haciendo una idea de lo que podía ser África" [J. L: Profesor de centro concertado urbano].

Dentro de los materiales educativos que se utilizan en el aula es posible distinguir las herramientas tecnológicas. Las herramientas tecnológicas aplicadas a la educación se caracterizan por su fácil adaptación y personalización, lo que permite que la tecnología se adapte a los estudiantes y sus necesidades y no a la inversa. En este sentido, el $53 \%$ del profesorado encuestado señala que su docencia a alumnos con discapacidad podría mejorar si dispusiese de las herramientas tecnológicas adecuadas $^{2}$. Aun así, el $65 \%$ de los centros a los que pertenecen los profesores encuestados no están equipados con tecnología específica que sirva de apoyo a los estudiantes con discapacidad.

\subsection{Apoyos de profesionales educativos}

El $95 \%$ de los profesores encuestados indicaron que los apoyos y colaboración con otros profesionales educativos ayudarían a mejorar su docencia orientada a alumnos con discapacidad. Más allá de la actividad que cada profesor desarrolla en su aula, los apoyos entre profesionales educativos "aumentan la capacidad de un centro para atender a la diversidad del alumnado" (Booth y Ainscow, 2002: I9). Como relató uno de los docentes entrevistados, la utilización de apoyos de especialistas en el aula implica la posibilidad de ampliar las oportunidades de aprendizaje de algunos alumnos:

2. En las entrevistas los docentes se han referido especialmente a las pizarras digitales, ordenadores en el aula y tecnología específica dirigida hacia las diferentes discapacidades, en concreto dispositivos de radiofrecuencia o lupas de aumento. 
“[...] Eso es lo que hacíamos con los ciegos, sobre todo. Entraba un profesor y se ponía al lado del chico o la chica invidente y le ayudaba un poco a entender lo que estábamos haciendo" [J. L.: Profesor de centro concertado urbano].

Pese a que el $69 \%$ de los docentes encuestados manifiesta que su centro cuenta con personal especializado para realizar tareas de apoyo, únicamente el $22 \%$ de los profesores estiman que cuentan con los apoyos necesarios y suficientes (Gráfico 4).

El apoyo debe interpretarse como la creación de una red de profesionales educativos en la que todos se benefician del conocimiento y experiencia de sus compañeros, "entendiendo y asumiendo el apoyo como una función inherente al desarrollo de la escuela, sin ubicarlo en unas personas o sector determinado o dirigido sólo a personas con unas características concretas o contextos específicos de intervención" (Gallego Vega, 20II: 94).

En consecuencia, para que los apoyos sean efectivos, es fundamental que la responsabilidad sobre la educación del alumno sea compartida, es decir, que el profesor de apoyo conozca la materia que imparte el profesor de la asignatura y, a su vez, este tenga información sobre el tipo de apoyo que está recibiendo el alumno con discapacidad (Liesa Hernández et al.,
20I2: 4I9). Los mundos de la especialización y la enseñanza normalizada, para los que tradicionalmente se han establecido itinerarios y formación diferentes deben "prepararse para una auténtica interacción y cooperación” (Núñez Mayán, 20IO: I7) pues de su sinergia se benefician todos los participantes en la actividad educativa. Como expresó uno de los profesores entrevistados:

\begin{abstract}
"Nosotros desde el colegio pedíamos más refuerzo, pedíamos algún refuerzo o apoyo específico de gente que estaba acostumbrada a trabajar con estos chicos. Y entonces que se pusiesen con ellos en momentos determinados a explicarles cosas que nosotros es que no terminábamos de llegar a que consiguiesen entender. Y ellos podían traer material de la ONCE específico y tal" [J.L.: Profesor de centro concertado urbano].
\end{abstract}

Es importante que los profesores sepan cuándo y cómo deben utilizar los apoyos de otros profesionales para evitar la etiquetación de los alumnos con discapacidad. Así, el dilema que deben resolver los distintos profesionales educativos para aplicar los apoyos correctamente es cómo compaginar la ayuda que recibe el alumno con dificultades para seguir la asignatura con el contexto ordinario de la clase (Moya Maya, 20I2). En este sentido, una de las claves para facilitar el éxito de los apoyos, cuando estos tienen lugar en el aula, es que el

Gráfico 4. Distribución del profesorado en función de si sus centros disponen de personal especializado que realiza tareas de apoyo

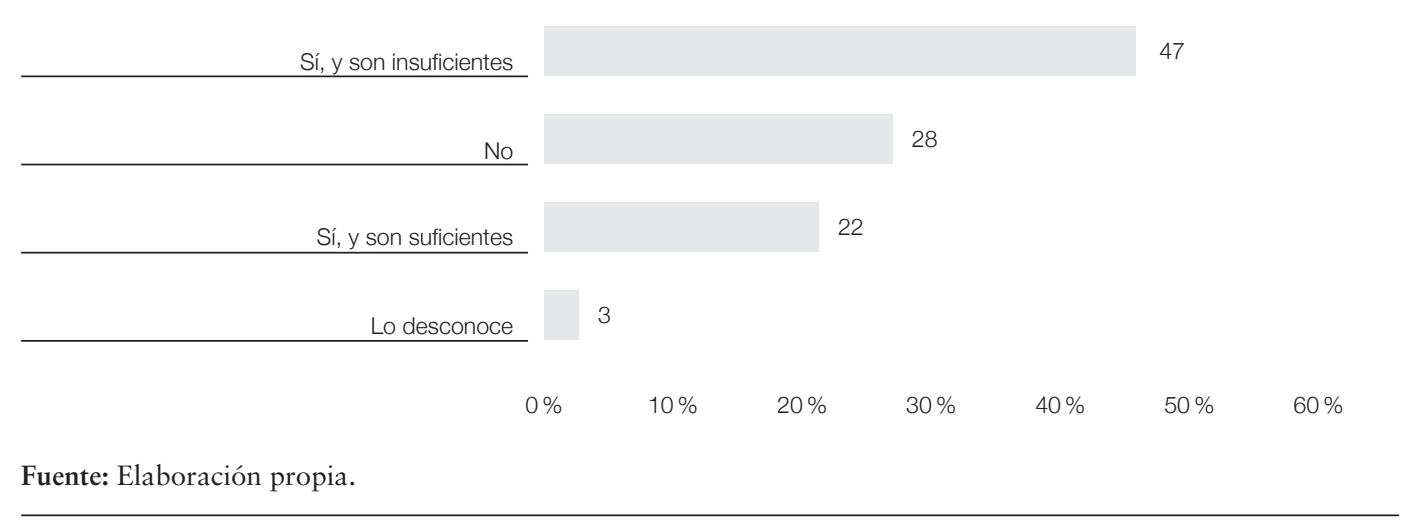


profesor genere un ambiente de normalidad y transmita a todo el grupo que el esquema tradicional de profesor y alumnos puede ser sustituido por dos o más profesores y los alumnos.

La colaboración entre los docentes es también importante para reflexionar y debatir, compartir experiencias y responsabilidades, superando de manera conjunta los retos y obstáculos que frecuentemente surgen en el proceso educativo. Como indicó una de las profesoras entrevistadas:

"como las problemáticas muchas veces van sucediendo, las reuniones son puestas en común por parte de los profesores: 'ah pues yo también lo tengo', 'ah pues yo creo que sería bueno...'. Al final siempre se toma la decisión 'vamos a ver si entre todos encontramos una solución...'. También creo que hablar con diferentes profesores de diferentes asignaturas y cursos nos ayuda a la resolución de conflictos" [C. D.: Profesora de centro concertado rural].

El 90\% de los profesores encuestados declararon que en su centro existe coordinación entre el profesorado, aunque en el $34 \%$ de los casos los docentes desarrollan una coordinación de carácter informal, sin el apoyo del centro educativo. Atendiendo a los diferentes tipos de centro se puede apreciar como los docentes que pertenecen a centros privados manifestaron tener menor coordinación entre el profesorado que sus compañeros de centros públicos y concertados (Tabla 4).

\subsection{Ratio profesor/alumno}

Tanto en las entrevistas realizadas a los docentes como en la información recabada mediante la encuesta, los profesores han hecho referencia a las dificultades que conlleva enseñar a grupos diversos con una atención individualizada, teniendo en cuenta las ratios tan elevadas de alumnos por aula. La correcta utilización de los distintos recursos educativos que posee el centro requiere que el profesorado identifique y comprenda las necesidades de todos los alumnos, pero para facilitar esta tarea es necesario que la ratio profesor/alumno sea modificada.

En este sentido, el $98 \%$ del profesorado encuestado declara que la ratio profesor/ alumno debe ser modificada para mejorar la docencia a alumnos con discapacidad. El profesorado entiende que en grupos reducidos las interacciones entre profesores y alumnos son mejores "de lo que serían en grupos grandes, lo que favorece cualitativa y cuantitativamente los aprendizajes" (Forteza y Sureda, 20I 2: 5). Además, es importante considerar que la reducción de la ratio beneficia fundamentalmente a aquellos alumnos más vulnerables, que tienen más dificultades para seguir las explicaciones del profesorado y realizar las actividades docentes. Una de las profesoras entrevistadas expresó su frustración por las ratios tan elevadas que tiene su centro de esta manera:

“¿qué le puedes dar de una sesión de cincuenta minutos? un minuto de tu atención...tienes que hacer una exposición de un tema, seguramente tienen dudas de tal, tienes que recoger, corregir Tabla 4. Distribución del profesorado en función de la coordinación con otros profesores del centro, por
tipo de centro

\begin{tabular}{|l|c|c|c|}
\hline Típos de coordinación & Público & Concertado & Privado \\
\hline No hay ninguna coordinación & $8 \%$ & $8 \%$ & $30 \%$ \\
\hline Sí, informalmente & $38 \%$ & $44 \%$ & $30 \%$ \\
\hline Sí, institucionalmente & $54 \%$ & $48 \%$ & $40 \%$ \\
\hline
\end{tabular}

Fuente: Elaboración propia. 
ejercicios... ¿qué puedes hacer con este chico que está ahí sentado?... pues atenderle brevemente, dar una vuelta a ver si se pierde... pero poco más... es que no hay más...” [N. Z : Profesora de centro público urbano].

El profesorado también puede verse afectado por tener que impartir docencia a grupos grandes y heterogéneos. La dificultad para poder atender a las necesidades de todos los alumnos y crear una dinámica positiva en el grupo puede afectar de manera determinante al estado de ánimo y al desarrollo de la actividad docente del profesorado (Rodríguez Tejada, Blázquez Entonado, Cubo Delgado, 2009: I 25 ). En este sentido se pronunciaron algunos de los profesores entrevistados:

"Pero lo que es un problema son las circunstancias actuales con grupos tan numerosísimos, eso sí es un problema. Y la sensación de impotencia que yo tengo por no poder atender a este chico adecuadamente" [E.M: Profesora de centro público rural].

Los profesores de centros públicos consideran, en mayor proporción que los profesores de centros concertados y privados que la ratio profesor/alumno debe ser modificada cuando en el grupo hay alumnos con discapacidad. Debido a que la presencia de alumnos con discapacidad es más frecuente en los centros públicos que en los concertados y privados, estos profesores se expresan, seguramente, desde el ámbito que aporta la experiencia y el conocimiento sobre las necesidades y los requerimientos que presenta un grupo cuando en él hay matriculados alumnos con discapacidad (Tabla 5).

\section{Discusión y conclusiones}

En las últimas décadas se ha producido una mejora evidente en la mayoría de los recursos educativos disponibles en los centros para que los docentes de secundaria atiendan a los estudiantes con discapacidad junto con el resto de sus compañeros (Casanova Rodríguez, 20II: I 5). No obstante, los profesores que han participado en nuestra investigación han manifestado que sus centros educativos carecen de todos los recursos educativos necesarios para desarrollar procesos educativos inclusivos.

Pese a que el planteamiento de la investigación tomó en consideración el ámbito y el tipo de centro el que trabajaban los profesores de secundaria los resultados han demostrado que las mayores diferencias en la disponibilidad de recursos educativos se encuentran en el carácter de los centros. Estos resultados son congruentes con los datos que aporta el Ministerio de Educación Cultura y Deporte (Tabla I), que muestra que la presencia de estudiantes con discapacidad en los centros educativos varía dependiendo del tipo de centro.

La desigual distribución de estudiantes y recursos educativos entre los centros escolares

Tabla 5. Distribución del profesorado según consideran la ratio profesor/alumno cuando se cuenta con estudiantes con discapacidad por tipo de centro

\begin{tabular}{|l|c|c|c|}
\hline $\begin{array}{l}\text { ¿Considera que el ratio profesor/alumno debe ser } \\
\text { diferente cuando se cuenta con estudiantes con } \\
\text { discapacidad? }\end{array}$ & Público & Concertado & Privado \\
\hline No & $1 \%$ & $1 \%$ & $10 \%$ \\
\hline Sí & $82 \%$ & $61 \%$ & $55 \%$ \\
\hline Sólo en algunos casos & $18 \%$ & $38 \%$ & $35 \%$ \\
\hline
\end{tabular}

Fuente: Elaboración propia. 
predispone a una segregación de los alumnos con discapacidad hacia aquellos centros educativos en los que reciben mejor atención y facilidades, generando una diversidad de ritmos en el desarrollo de la educación inclusiva (Verdugo Alonso y Rodríguez Aguilella, 2010: 465-466; Toboso Martín et al, 20I 2: 292). Esto supone una brecha entre los planteamientos teóricos sobre la educación a estudiantes con discapacidad, que promulgan una escuela para todos, y lo que actualmente refleja el sistema educativo español en la etapa de educación obligatoria.

En particular, la insuficiencia o insatisfacción con los recursos educativos existentes en muchos centros indica la distancia que actualmente separa la teoría de la práctica inclusiva. En este sentido, la descoordinación entre los propósitos y los resultados llevó a algunos de los profesores entrevistados a expresarse de esta manera tan elocuente:

\footnotetext{
"hay que ocuparse de todo el mundo, entonces... Si, lo ideal es mantener un equilibrio, pero claro, lo que no se puede hacer es abandonar a nadie...y...si al final uno ve que en el centro no existen los recursos suficientes, las posibilidades de atender ese caso pues hay que intentar derivarlo a un sitio donde creamos que sí que...eso puede ocurrir...” [R.R.: Profesor de centro público urbano] Es este su sitio?
}

Para analizar la desigual distribución de recursos y estudiantes con discapacidad entre los centros educativos es preceptivo tener también en consideración la paradoja que se produce cuando determinados centros se proveen de recursos para atender a estudiantes con discapacidad. Es posible que el resultado del progreso inclusivo de ciertos centros transgreda los mismos principios que pretende alcanzar: la inclusión. Los centros educativos con mayores recursos se pueden convertir en un reclamo para otros estudiantes con discapacidad y prolongar, involuntariamente, la segregación de los centros educativos del pasado, que los diferenciaba entre normales y especiales o alternativos al aula ordinaria (Moliner García, 2008: 34). Además de invertir en mejores escuelas hay que hacerlo en escuelas en las que todo el alumnado pueda educarse de manera conjunta, mejorando la calidad educativa de la escuela regular "de modo que sea posible una escuela para todos en la que todos los alumnos sean solamente eso, alumnos" (Ainscow, Echeita Sarrionandia y Duk, I994: 7).

Gerardo Echeita Sarrionandia (2013: I09) cuestiona con ironía si los derechos de los estudiantes con discapacidad a recibir una educación inclusiva es un derecho dependiente de la existencia de recursos educativos en el centro al que quieren acudir. La desigual presencia de recursos en los centros educativos no solo crea una desigualdad en la atención a los estudiantes con discapacidad, también vulnera uno de los principios fundamentales de la escuela inclusiva: que los estudiantes acudan al centro más cercano a su domicilio junto al resto de sus compañeros. En este sentido, el mismo autor, Gerardo Echeita, en un artículo titulado “¿Por qué Jorge no puede ir al mismo colegio que su hermano?” (2004) describe un hecho habitual en los centros escolares españoles. Jorge es un estudiante con síndrome de Down que puede ir al mismo colegio que su hermano, la legislación vigente le ampara, pero el contexto educativo, los profesionales educativos y en última instancia los padres, desilusionados con su idea inicial, hacen que Jorge termine segregado en un centro de educación especial. Uno de los aspectos que más llaman la atención de la situación descrita es que todos los actores terminan convencidos de que sin ser una opción idónea, es la mejor solución para Jorge.

La falta de recursos para atender a todo el alumnado en los centros educativos de secundaria es un serio obstáculo al que se añade la ausencia de criterios homogéneos que garanticen, con un estricto cumplimiento, que los alumnos con discapacidad no serán derivados a centros especializados. Se trata de una opción que pese a ser hoy en día minoritaria, pues solo el $2 \%$ de los profesores encuestados en esta investigación ha indicado que es la forma de actuar de su centro, todavía tiene lugar (Gráfico I).

No obstante, un elemento que puede resultar esperanzador en cuanto al desarrollo actual 
de procesos inclusivos es la actitud que hemos detectado en los docentes que han participado en nuestra investigación. El 9I \% de los docentes encuestados opinaron que los alumnos con discapacidad deberían recibir apoyos especializados en el mismo centro, evitando la segregación a otros centros en los que los alumnos que no son considerados "normales" reciben una formación independiente. Aunque esta es sin duda una percepción inclusiva sobre la educación, es necesario mencionar la interpretación que algunos docentes hacen de los apoyos de especialistas y que fundamentalmente hemos identificado a través de las entrevistas en profundidad. Se trataría del tipo de apoyo que genera "itinerarios paralelos" o procesos de exclusión interna que provocan que los alumnos con discapacidad se eduquen en el mismo centro que sus compañeros pero separados espacial o temporalmente de ellos, lo que puede producirse si los alumnos reciben los apoyos en estancias diferentes o realizan actividades distintas en el mismo aula (Martínez Domínguez, 2005; Young, 2000). En definitiva, las entrevistas en profundidad nos han mostrado a un profesorado que, pese a no conocer en todos los casos los principios y actuaciones adecuadas para que los estudiantes con discapacidad reciban una educación inclusiva, se muestra, en términos generales, voluntarioso y con una actitud positiva para que todos los estudiantes se puedan educar de manera conjunta.

Un segundo aspecto que resulta alentador es la propia comprensión de la escuela inclusiva como un proceso y no un producto (Echeita Sarrionandia, 2007). Pese a que los recursos educativos para atender a los estudiantes con discapacidad no son hoy en día totalmente satisfactorios, el desarrollo de ideas y actitudes inclusivas entre los distintos profesionales educativos indica que las políticas y proyectos educativos están lentamente asentándose entre la comunidad educativa. Las políticas y proyectos inclusivos están ligados a la provisión de recursos educativos y pese a tratarse elementos intangibles, no siempre perceptibles, son fundamentales para articular el resto de recursos.

Así, la existencia de personal que preste apoyo a los alumnos con discapacidad puede suponer una ayuda en su educación si estos se utilizan correctamente y cuando son necesarios pero, también, puede suponer un obstáculo y fomentar su exclusión si los apoyos implican la segregación o etiquetaje del estudiante cuando su educación sucede en tiempos o estancias diferentes al resto de la clase. Algo similar ocurre con la disminución de las ratios. Disminuir el número de estudiantes por grupo-clase puede suponer una relación más cercana con los estudiantes, mayor posibilidad de conocer las necesidades del alumno y un elemento que beneficia la dinámica interna del grupo. Pero también, los grupos más pequeños pueden conducir a clasificar y diferenciar a los alumnos si estos se configuran atendiendo a las características y capacidades del alumnado. Como indicó una docente entrevistada:

“...es importante la ratio, pero también es importante la organización pedagógica de la clase...es decir, la ratio por sí misma es un elemento importante, pero si no hay luego una organización pedagógica adecuada..." [V. M: Profesora de centro público urbano].

En definitiva, para desarrollar procesos inclusivos, además de la presencia de los recursos en el centro escolar, es fundamental que se haga un uso apropiado de los mismos por parte del personal educativo y que todos estos recursos estén acompañados de una cultura y valores que sirvan para que los estudiantes con discapacidad puedan tener presencia y participar en el centro educativo.

La política y cultura del centro afecta a los valores sobre los que se asienta la docencia y la relación entre los diferentes actores presentes en el proceso educativo. En este sentido, una sociedad diversa y heterogénea debe ser capaz de educar a sus miembros desde la diversidad y no solo sobre ella, trasladando unos principios y valores de la teoría a la práctica. Pese a tratarse de principios asumidos por una gran mayoría de la población, la realidad de los centros educativos nos han mostrado que todavía, atendiendo a la experiencia de los profesores que trabajan en ellos, este escenario no siempre es posible. 
Ainscow, M., Echeita Sarrionandia, G. y Duk, C. (I994). "Necesidades Especiales en el Aula. Una iniciativa de la UNESCO para la formación del profesorado en el ámbito de la integración escolar". Aula de innovación educativa, 2(3 I), 70-77.

Ainscow, M. (1995). Necesidades especiales en el aula: Guí para la formación del profesorado. Madrid: Narcea Ediciones.

Ainscow, M. Booth, T y Dyson, A. (2006). Improving schools, developing inclusion, Londres: Routledge.

Alonso Parreño, M. J. y Araoz Sánchez-Dopico, I. (2OII). El impacto de la Convención Internacional sobre los Derechos de las Personas con Discapacidad en la legislación educativa española. Madrid: Ediciones Cinca (en línea). <http://www. convenciondiscapacidad.es/ColeccionONU_ new/Impacto \% 2ode \% 2ola \% 20C.6.pdf>. Acceso el 6 de mayo de 2014 .

Booth, T. y Ainscow, M. (2002). Guía para la evaluación y mejora de la educación inclusiva: desarrollando el aprendizaje y la participación en las escuelas, Madrid: Consorcio Universitario para la Educación Inclusiva, Universidad Autónoma de Madrid.

Caballero Méndez, F. y Díaz Gandasegui, V. (20I4). Las necesidades formativas de los docentes en la educación de estudiantes con discapacidad, Madrid: Fundación ONCE.

Casanova Rodríguez, M. A y Cabra de Luna, M. A. (2009) Educación y personas con discapacidad: presente y futuro, Madrid: ONCE.

Casanova Rodríguez, M. A. (20I I), "De la educación especial a la inclusión educativa. Estado de la cuestión y retos pendientes”, CEE Participación Educativa, I 8, pp. 8-24.

Díaz Lucea, J. (I996). "Los recursos y materiales didácticos en Educación Física”, Apunts: Educación física y deportes, (43), 42-54 (en línea). <http://dialnet.unirioja.es/servlet/ articulo? codigo $=296386>$.
Echeita Sarrionandia, G. (2004). “¿Por qué Jorge no puede ir al mismo colegio que su hermano? Un análisis de algunas barreras que dificultan el avance hacia una escuela para todos y con todos". REICE Revista Electrónica Iberoamericana sobre Calidad, Eficiencia y Cambio en Educación, 2(2).

Echeita Sarrionandia, G. (2007). Educación para la exclusión o educación sin exclusiones, Madrid: Narcea.

Echeita Sarrionandia, G. (2009). "Los procesos de inclusión educativa desde la Declaración de Salamanca. Un balance entre doloroso y esperanzado", en Giné, C. (coord.): La educación inclusiva. De la exclusión a la plena participación de todo el alumnado. Barcelona: Horsori.

Echeita Sarrionandia, G. (20I3). "Inclusión y exclusión educativa. De nuevo. Voz y quebranto". REICE. Revista Iberoamericana sobre Calidad, Eficacia y Cambio en Educación, II(2), 99-I I 8.

Ferrandis, M. V., Grau, C. y Fortes, M. C. (2010). "El profesorado y la atención a la diversidad en la ESO”. Revista de Educación Inclusiva, 3(2), I I-28.

Ferreira, M. A. (20I3). "La brecha entre la normativa y la experiencia subjetiva: políticas públicas, discapacidad y crisis económica”. En IV Congreso REPS, Universidad de Alcalá, 6 y 7 de Junio de 20I3. (en línea). <http://www3.uah.es/congresoreps20I3/ Paneles/panel4/sesionI/mavferre@ucm.es/ TC2013REPStextocompleto.pdf.>

Forteza, D. y Sureda, J. (20I2). "Sobre la ratio de alumnos por aula”. Dosier de Actualidad, Departamento de Pedagogía Aplicada y psicología de la Educación, Universidad de Illes Balears, I (en línea). <http://pape.uib.es/sites/ default/files/files/dossier\% $\%$ oactualidad $\%$ 2oES. pdf $>$. Acceso el 7 de febrero de 2013 .

Gallego Vega, C. (20I I). "El apoyo inclusivo desde la perspectiva comunitaria”, Revista 
Interuniversitaria de Formación del Profesorado, 70 (25, I), 93-109 (en línea). $<$ http://aufop.com/aufop/uploaded_files/ revistas/I342I289293.pdf>.

García Pastor, C. (2005). Educación y diversidad. Málaga: Aljibe.

García Romero, C. (2004). "La teoría y la práctica de la evaluación y el informe psicopedagógicos". Tavira: Revista de ciencias de la educación, (20), 45-58.

Liesa Hernández, E., Castelló, M., Carretero Torres, M. R., Cano, M., Mayoral, P. (2OI2). "La atención a la diversidad en la educación secundaria obligatoria: valorciones de los profesionales". Profesorado. Revista de Currículum y Formación de Profesorado, vol. I6, núm. 2, 403-420.

Martínez Domínguez, B. (2005). "Las medidas de respuesta a la diversidad. Posibilidades y límites para la inclusión escolar y social”. Profesorado, Revista de Currículum y Formación del Profesorado, I, (I) I-3I.

MEC (I996). La evaluación psicopedagógica: modelo, orientaciones, instrumentos. Madrid: MEC.

Moliner García, O. (2008). “Condiciones, procesos y circunstancias que permiten avanzar hacia la inclusión educativa: retomando las aportaciones de la experiencia canadiense”. REICE-Revista Electrónica Iberoamericana sobre Calidad, Eficacia y Cambio en Educación, 6(2).

Moya Maya, A. (20I2). "El profesorado de apoyo en los centros ordinarios”. Nuevas funciones, nuevas contradicciones, Educatio Siglo XXI, $30(\mathrm{I})$.

Núñez Mayán, M. T. (20I0). "La formación del profesorado para la inclusión educativa”. II Congreso Iberoamericano de Síndrome de Down, Granada, 29 de Abril al 2 de Mayo de 20 Io (en línea). <http://www. granadazoIodown.org/adjuntos/cTexto/9_I_ congreso_o.pdf>.

Parrilla Latas, A. (2009).” ¿Y si la investigación sobre inclusión no fuera inclusiva?” Reflexiones desde una investigación biográfico-narrativa. Revista de Educación, 349, IOI-I I7.

Porter, G. L. (I997). "Crirical elements for inclusive schools”. En Pijl, S.J; Meijer, C. J. W. y Hegerty, S. Critical Elements for Inclusive Schools, Londres: Routledge.

Rodríguez Tejada, R. M., Blázquez Entonado, F. y Cubo Delgado S. (2009). "Profesores de ESO y necesidades educativas especiales”. Enseñanza, 25, III-I 34 .

Sandoval Mena, M., Simón Rueda, C. y Echeita Sarrionandia, G. (2OI2). "Análisis y valoración crítica de las funciones del profesorado de apoyo desde la educación inclusiva". Revista de Educación, número extraordinario, I I7-I37.

Téllez Gallego, M. I. (20I0). "Detección temprana de alumnos/as con necesidades específicas de apoyo educativo". Innovación y experiencias educativas, 26 (en línea). <http://www.csi-csif. es/andalucia/modules/mod_ense/revista/pdf/ Numero_26/M_ISABEL_TELLEZ_I.pdf>. (Consultado el Io/O2/20I3).

Toboso Martín, M., Ferreira, M. A., Díaz Velázquez, E., Fernández-Cid Enríquez, M. C., Villa Fernández, N. y Gómez de Esteban, C. (20I2). "Sobre la educación inclusiva en España: políticas y prácticas”. Intersticios. Revista sociológica de pensamiento crítico, 6(I).

Trujillo Torres, J.M, Torres González, J.A., Hernández Fernández, A. (20I I). "Los recursos materiales y personales para la atención de los alumnos y de las alumnas con necesidades educativas especiales". Revista internacional de educación, tecnologías de la información y comunicación aplicadas a la educación inclusiva, logopedia y multiculturalidad, I(I), 82 .

Vallejo Ruiz, M.y Bolarín Martínez, M. J. (20I I). "Los programas de atención a la diversidad y los centros de educación secundaria: claves organizativas, recursos, adscripción del profesorado y valoración de los programas por el profesorado". Profesorado. Revista de curriculum y formación del profesorado. I3(3), I 43 -I 55 . 
Verdugo Alonso, M. A. y Rodríguez Aguilella, A. (2010). "La inclusión educativa en España desde la perspectiva de alumnos con discapacidad intelectual, de familias y de profesionales". Revista de educación, 358 , 450-470.

Young, I. M. (2000). Inclusion and Democracy. Oxford: Oxford University Press. 